\title{
Transforming growth factor $\beta 1$ enhances stemness of head and neck squamous cell carcinoma cells through activation of Wnt signaling
}

\author{
WOO-JIN BAE ${ }^{1 *}$, SANG-HYUK LEE ${ }^{2 *}$, YOUNG-SOO RHO ${ }^{3}$, BON-SEOK KOO ${ }^{4}$ and YOUNG-CHANG LIM ${ }^{1}$ \\ ${ }^{1}$ Department of Otorhinolaryngology-Head and Neck Surgery, Research Institute of Medical Science, \\ Konkuk University School of Medicine, Seoul 142-702; ${ }^{2}$ Department of Otorhinolaryngology-Head and Neck Surgery, \\ Sungkyunkwan University School of Medicine, Kangbuk Samsung Hospital, Seoul 03181; \\ ${ }^{3}$ Department of Otorhinolaryngology-Head and Neck Surgery, Ilsong Memorial Institute of Head and Neck Cancer, \\ Hallym University, College of Medicine, Seoul 05355; ${ }^{4}$ Department of Otorhinolaryngology-Head and Neck Surgery, \\ Chungnam National University, College of Medicine, Daejeon 35015, Republic of Korea
}

Received April 19, 2015; Accepted September 22, 2016

DOI: $10.3892 / 01.2016 .5336$

\begin{abstract}
Transforming growth factor $\beta$ (TGF $\beta$ ) ligands, including TGF $\beta 1$, are multifunctional cytokines known as key regulators of cell growth, differentiation and inflammation. Dysregulated TGF $\beta$ signaling is common in numerous solid tumors, including head and neck squamous cell carcinoma (HNSCC). Previously, TGF $\beta$ ligands were also reported to be associated with an enhancement of stemness in glioma stem-like cells. However, their role in HNSCC cancer stem cells (CSCs) has not been explored. The present study demonstrated that TGF $\beta 1$ enriches the properties of HNSCC CSCs. TGF $\beta 1$ promoted sphere formation and increased stemness-associated gene expression (Oct4 and Sox2) of primary HNSCC CSCs. Additionally, the population of aldehyde dehydrogenase (ALDH)-positive cells was increased subsequent to exogenous treatment of cells with TGF $\beta 1$. In addition, following stimulation with TGF $\beta 1$, the cells exhibited more resistance to cisplatin and elevated expression of Twist, Snail and Slug. Mechanistically, TGF $\beta 1$ acts as an upstream stimulator of Wnt/ $\beta$-catenin signaling. Collectively, the present findings provide insights toward the development of TGF $\beta 1$ signaling inhibition strategies for treating HNSCC CSCs.
\end{abstract}

Correspondence to: Professor Young Chang Lim, Department of Otorhinolaryngology-Head and Neck Surgery, Research Institute of Medical Science, Konkuk University School of Medicine, 120-1 Neungdong-ro (Hwayang-dong), Gwnagjin-gu, Seoul 142-702, Republic of Korea

E-mail: yclim69@hanmail.net

*Contributed equally

Key words: head and neck squamous cell carcinoma, cancer stem cells, TGF $\beta 1$

\section{Introduction}

Head and neck squamous cell carcinoma (HNSCC) is the sixth most common cancer worldwide and has a poor prognosis (1). The overall survival rate has not changed over the past 30 years, with $>400,000$ patients diagnosed and >200,000 mortalities annually (2). Despite numerous advances in treatment modalities and techniques, including surgery, chemotherapy, radiation therapy and combinations of these, only 50-60\% of patients survive for $>5$ years (3). The static survival rate is considered to be the result of a high incidence of treatment resistance, resulting in local/regional recurrences and frequent distant metastases (4). Thus, to improve the survival of patients with HNSCC, an increased understanding of the pathophysiology and tumorigenesis of HNSCC is urgently required.

A previous study revealed that a small subpopulation of tumor cells, cancer stem cells (CSCs), can initiate and maintain tumors and metastasize (5). As conventional therapies usually target differentiated cancer cells, CSCs are considered to be responsible for treatment failure (6). Thus, a considerable understanding and identification of the promoters and regulators that control expression of HNSCC CSCs may lead to methods to increase the prognosis of HNSCC against treatment failure.

Transforming growth factor $\beta$ (TGF $\beta$ ) is a well-known and potent regulator of the extracellular matrix (7). In human cancer, TGF $\beta$ shows biphasic roles in tumor development and progression (8). TGF $\beta$ functions as a tumor suppressor by cellular proliferation inhibition or by promoting differentiation of normal cells (9). Mutations of type II receptors in the TGF $\beta$ signaling pathway have been demonstrated for head and neck cancer (10). However, once tumor cells become resistant to the proliferation-inhibiting function of TGF $\beta$, they increase their production of TGF $\beta$. As a result, the increase in TGF $\beta$ leads to TGF $\beta$-mediated angiogenesis stimulation, immune suppression and cell motility stimulation (11). Consequently, an increased TGF $\beta$ level results in increased invasiveness of tumor cells. Previously, it was reported that TGF $\beta$ is able to 
increase the proportion of cluster of differentiation (CD) $133^{+}$(a putative marker of CSCs) cells in liver cancer cells, suggesting a positive role for TGF $\beta$ in the 'stemness' of cancer cells (12). However, whether TGF $\beta$ stimulates the key features of this cell subpopulation in HNSCC has not been reported.

In the present study, the effect of an increased TGF $\beta$ level on the stemness of HNSCC CSCs was investigated. The association with the $\mathrm{Wnt} / \beta$-catenin signaling pathway was also examined to elucidate the downstream effect of TGF $\beta$ on stemness regulation in HNSCC CSCs.

\section{Materials and methods}

Cell culture and reagents. The primary sphere K3 cell line was used. This cell was isolated from a surgical specimen from a HNSCC patient, and the CSC properties of the cell line have been validated using a number of functional assays, testing self-renewal capability, stem cell marker expression, chemoresistance and in vivo tumorigenicity, as previously reported (5). Cells were cultured in serum-free Dulbecco's modified Eagle's medium (DMEM)-Ham's F-12 (F12) medium supplemented with human recombinant basic fibroblast growth factor (bFGF; 10 ng/ml; R\&D Systems, Minneapolis, MN, USA), N2 supplement ( $1 \mathrm{ml}$ per $500 \mathrm{ml}$ medium; Gibco; Thermo Fisher Scientific, Inc., Waltham, MA, USA), and epidermal growth factor (EGF; $10 \mathrm{ng} / \mathrm{ml}$; R\&D Systems). The primary $\beta$-catenin antibody was purchased from Santa Cruz Biotechnology, Inc. (Dallas, TX, USA), and the secondary anti-mouse IgG antibody was obtained from Jackson ImmunoResearch Laboratories (West Grove, PA, USA).

Tumorsphere formation assay. To assess self-renewal in vitro, the cells were dissociated into a single-cell suspension, seeded in a 24 -well plate at a density of 500 cells per well, and cultured in serum-free medium, with EGF and bFGF added every other day. Untreated cells (cells not treated with EGF or bFGF) were used as a control. Tumorspheres were allowed to grow for 7 days, and spheres with a diameter $>30 \mu \mathrm{m}$ were counted.

Reverse transcription-quantitative polymerase chain reaction (RT-qPCR) and western blotting. RT-qPCR and western blotting experiments were performed as previously described (5). RT-qPCR analysis of Wnt signaling target genes ( $\beta$-catenin, c-myc and cyclin D) was subsequently performed on an iCycler IQ real-time detection system (Bio-Rad Laboratories, Hercules, CA, USA), using IQ Supermix with SYBR-Green (Bio-Rad Laboratories). The sequences of the human-specific primers used were as follows: Oct4 forward, 5'-GCAATT TGCCAAGCTCCTGAA-3' and reverse, 5'-GCAGATGGT CGTTTGGCTGA-3'; Sox 2 forward, 5'-CCTCCGGGACAT GATCAG-3' and reverse, 5'-TTCTCCCCCCTCCAGTTC-3'; Twist forward, 5'-CAGTCTTACGAGGAGCTGCA-3' and reverse, 5'-TCTTGCTCAGCTTGTCCGAG-3'; Snail forward, 5'-CACCTCCAGACCCACTCAGAT-3' and reverse, 5'-CCT GAGTGGGGTGGGAGCTTC-3'; Slug forward, 5'-CTCCAT GCCTGTCATACCAC-3' and reverse, 5'-GGAAAGAGG AGAGAGGCCAT-3'; $\beta$-catenin forward, 5'-GGCAGTGCG TTTAGCTGGTG-3' and reverse, 5'-AGCTTGGGGTCCACC ACTAG-3'; c-myc forward, 5'-GTATGTGGACGGCTTCTC
GC-3' and reverse, 5'-GCTGCTGTCGTTGAGAGGGT-3'; and cyclin D forward, 5'-GGAGCTGCTGCAAATGGAGC-3' and reverse, 5'-CAGAGGGCAACGAAGGTCTG-3'.

Aldehyde dehydrogenase $(A L D H)$ activity assay. ALDH activity was analyzed using an Aldefluor assay kit (Stem Cell Technologies, Vancouver, BC, Canada). Briefly, cells were suspended in Aldefluor assay buffer containing the ALDH substrate $\left(1 \mu \mathrm{mol} / 1\right.$ per $1 \times 10^{6}$ cells $)$. A sample of cells was stained with the specific ALDH inhibitor diethylaminobenzaldehyde (DEAB) as a negative control. Fluorescent $\mathrm{ALDH}^{+}$ cells were detected in the green fluorescence channel, and samples treated with the specific ALDH inhibitor DEAB were used as the control to set the gates defining the $\mathrm{ALDH}^{+}$region. Flow cytometric sorting was conducted using a FACSAria flow cytometer (Becton Dickinson, San Jose, CA, USA).

Chemosensitivity assay. Cells were dissociated into a single-cell suspension and then plated in a 24 -well plate at a density of $1 \times 10^{5}$ cells per well under serum-free culture conditions. The cells were treated with cisplatin at the indicated concentrations and then cultured at $37^{\circ} \mathrm{C}$ in a humidified $5 \%$ $\mathrm{CO}_{2}$ atmosphere. Subsequently, $24 \mathrm{~h}$ later, $50 \mu \mathrm{l}$ of MTT solution $(5 \mathrm{mg} / \mathrm{ml}$ in PBS) was added to each well, and the plate was incubated at room temperature for $2 \mathrm{~h}$. Absorbance was measured on a SpectraMax 190 device (Molecular Devices, LLC, Sunnyvale, CA, USA) at a wavelength of $570 \mathrm{~nm}$.

Optimal Tcf-binding site (TOP)/far-from-optimal Tcf-binding site (FOP) luciferase assay. Cells were seeded in 24-well plates at a density of $1 \times 10^{5}$ cells per well. Cells were transiently transfected using Polyexpress ${ }^{\circledR}$ (Excellgen, Rockville, MD, USA). For the luciferase assay, cells were transfected with $0.5 \mu \mathrm{g}$ of the lymphoid enhancer factor-reporter pTOPFLASH/pFOPFLASH along with $50 \mathrm{ng}$ of pRL-SV40. TGF $\beta 1$ treatment was initiated at $24 \mathrm{~h}$ post-transfection and performed for $48 \mathrm{~h}$. Luciferase activity was monitored using the Dual-Glo luciferase assay system (Promega Corporation, Madison, WI, USA). The transfection efficiency was normalized to the cotransfected Renilla luciferase activity according to the manufacturer's protocol.

Statistical analysis. Experimental data were statistically assessed using either 2-tailed Student's t-test or analysis of variance with Scheffe's post-hoc analysis. $\mathrm{P}<0.05$ was considered to indicate a statistically significant difference.

\section{Results}

TGF $\beta 1$ increases the self-renewal capacity of cancer stem cells. The capacity for self-renewal is one of the putative traits of CSCs (5). Thus, it was evaluated whether TGF $\beta 1$ treatment enhances the self-renewal capacity of HNSCC CSCs by performing a sphere-forming assay. TGF $\beta 1(2 \mathrm{ng} / \mathrm{ml})$ treatment significantly increased the sphere formation ability of HNSCC CSCs with a diameter of $>30 \mu \mathrm{m}$ compared to the control treatment group (Fig. 1).

TGF $\beta 1$ increases the levels of stemness markers of CSCs. Oct 4 and Sox 2 are critical regulators of pluripotency in the 
A

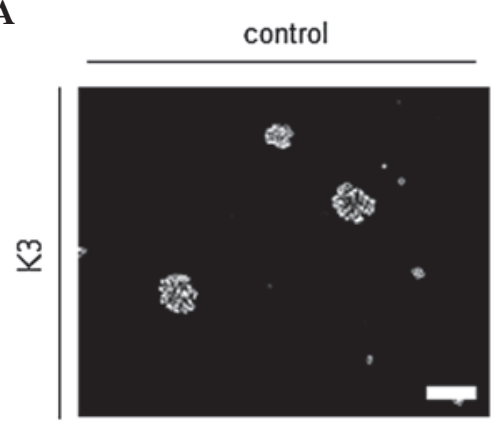

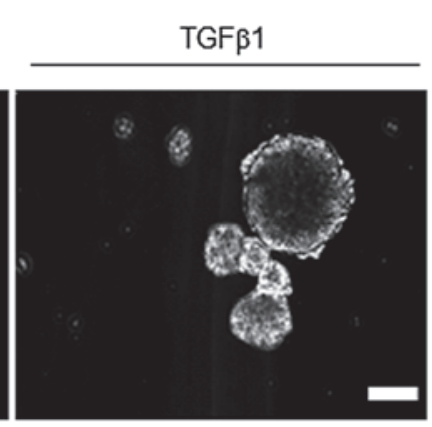

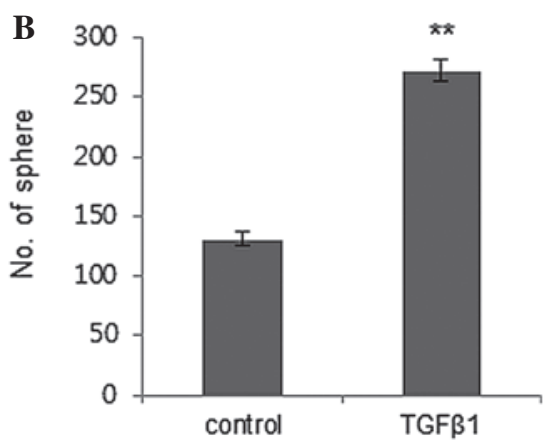

Figure 1. TGF $\beta 1$ treatment increases the sphere-forming ability of HNSCC K3 CSCs. (A) Photomicrographs of representative spheres after exogenous TGF $\beta 1$ or control (dimethyl sulfoxide) treatment in HNSCC CSCs, (B) with quantification. Scale bar, $30 \mu \mathrm{M}$. ${ }^{* *} \mathrm{P}<0.01$. TGF $\beta 1$, transforming growth factor $\beta ;$ HNSCC, head and neck squamous cell carcinoma; CSCs, cancer stem cells.

A

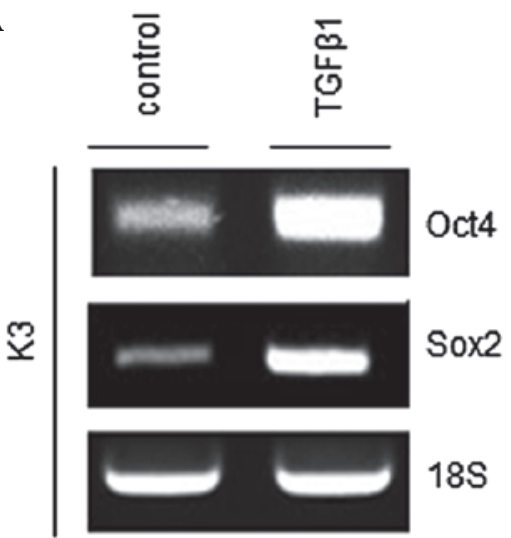

B
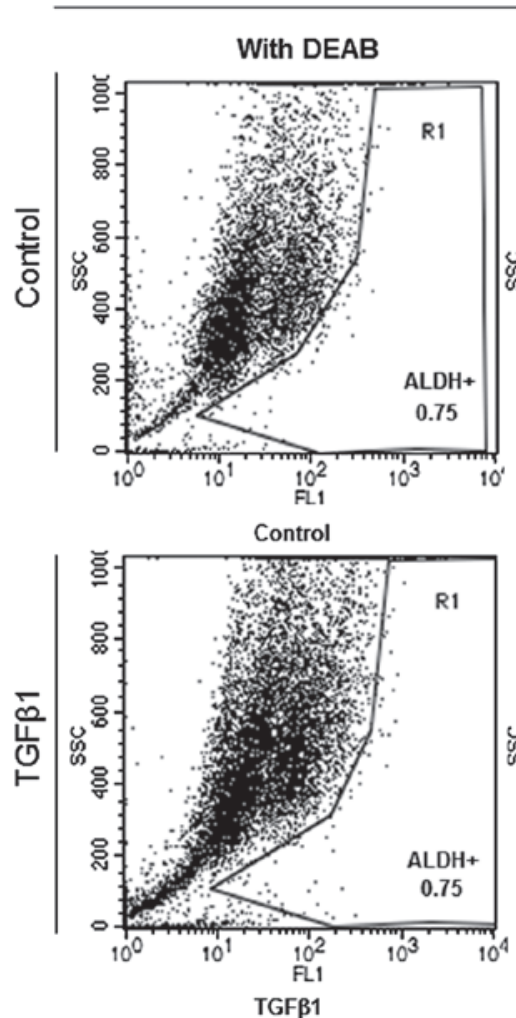

K3
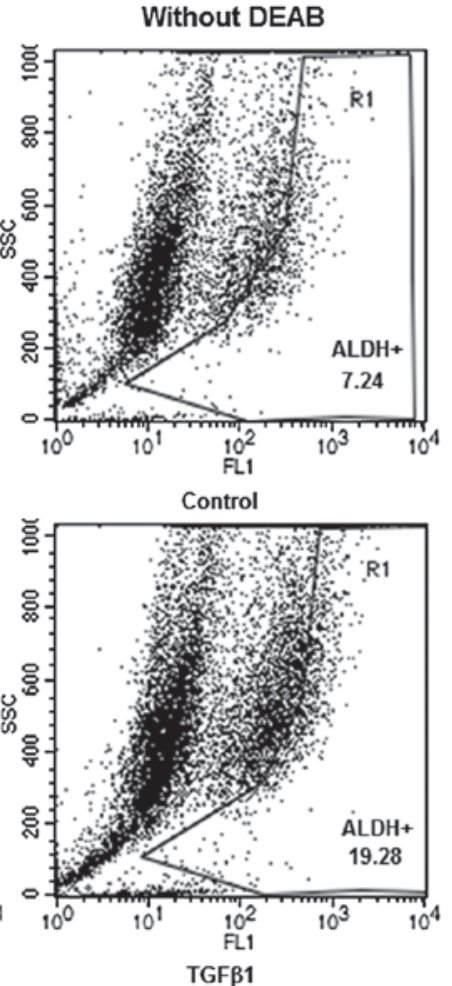

Figure 2. TGF $\beta 1$ treatment increases stemness-associated marker expression in HNSCC CSCs. (A) Transcript levels of Oct4 and Sox 2 in TGF 1 - or control (DMSO)-treated HNSCC CSCs. (B) ALDH activity subsequent to TGF $\beta 1$ or control (DMSO) treatment of HNSCC CSCs. TGF $\beta 1$, tumor growth factor $\beta$; HNSCC, head and neck squamous cell carcinoma; CSCs, cancer stem cells; DMSO, dimethyl sulfoxide; DEAB, diethylaminobenzaldehyde.

mammalian embryo, and deregulated expression of these genes can be found in HNSCC CSCs (13). Thus, whether TGF $\beta 1$ treatment increases the transcriptional expression of Oct 4 and Sox 2 was examined. The results showed that mRNA expression levels of Oct4 and Sox 2 were significantly increased in the TGF $\beta 1$ treatment group compared to the control treatment group (Fig. 2A). ALDH is considered to be a marker of HNSCC CSCs (14). Therefore, ALDH activity was examined in TGF $\beta 1$ - and control-treated HNSCC CSCs. Fig. 2B shows that the proportion of $\mathrm{ALDH}^{+}$cells was significantly increased in the TGF $\beta 1$ treatment group compared with the control treatment group (7.24 vs. $19.28 \%)$.
TGF $\beta 1$ increases chemoresistance to cisplatin for HNSCC CSCs by overexpression of ATP-binding cassette sub-family $G$ member 2 (ABCG2). HNSCC CSCs possess a chemoresistance ability against anticancer drugs, and the ABCG2 transporter is responsible for this resistance phenotype (5). Therefore, chemoresistance levels were compared using MTT assay subsequent to cisplatin administration at various concentrations with the TGF $\beta 1$ and control treatment groups. The results showed that TGF $\beta 1$-treated HNSCC CSCs had significantly increased cisplatin resistance compared with control-treated HNSCC CSCs (Fig. 3A). Furthermore, an increased $\mathrm{ABCG} 2$ expression level was identified in TGF 1 1-treated HNSCC CSCs compared to control-treated 

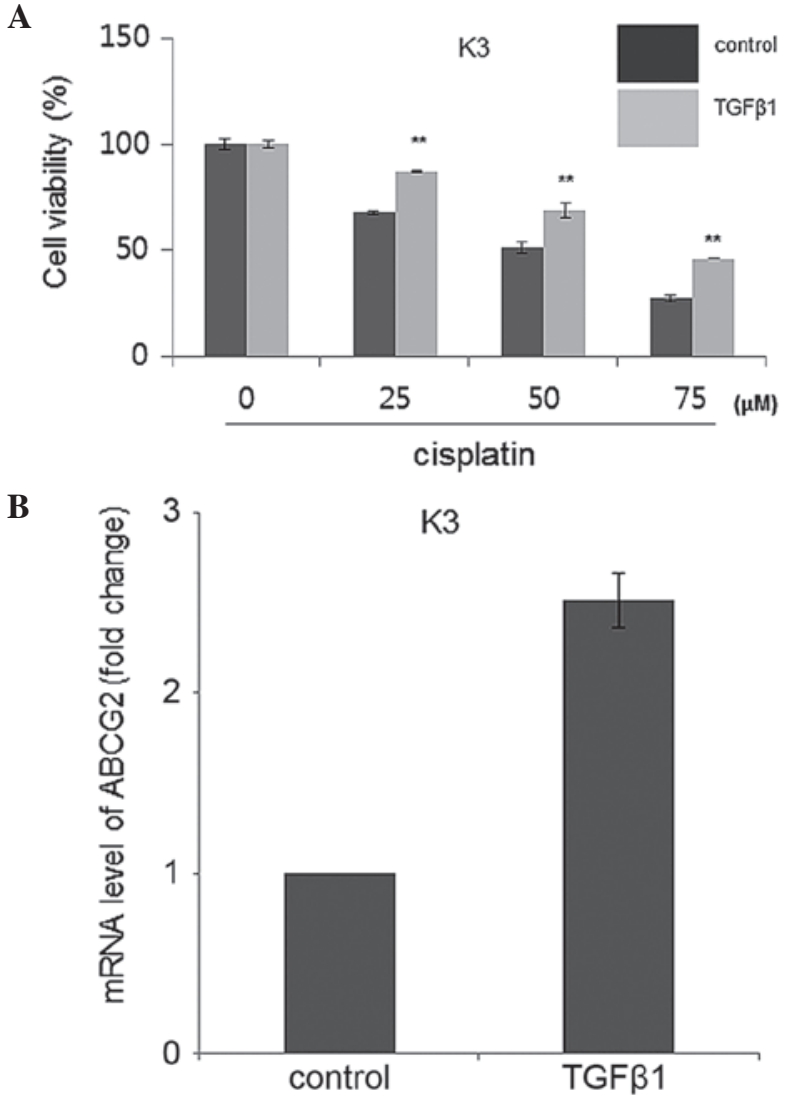

Figure 3. TGF $\beta 1$ treatment enhances cisplatin resistance of HNSCC K3 CSCs. (A) Cell viability subsequent to cisplatin administration at various concentrations of TGF $\beta 1$ - or control (DMSO)-treated HNSCC CSCs. (B) mRNA levels of ABCG2 in TGF $\beta 1$ - or control (DMSO)-treated HNSCC CSCs. ${ }^{* *} \mathrm{P}<0.01$. TGF $\beta 1$, transforming growth factor $\beta$; HNSCC, head and neck squamous cell carcinoma; CSCs, cancer stem cells; DMSO, dimethyl sulfoxide; ABCG2, ATP-binding cassette sub-family G member 2

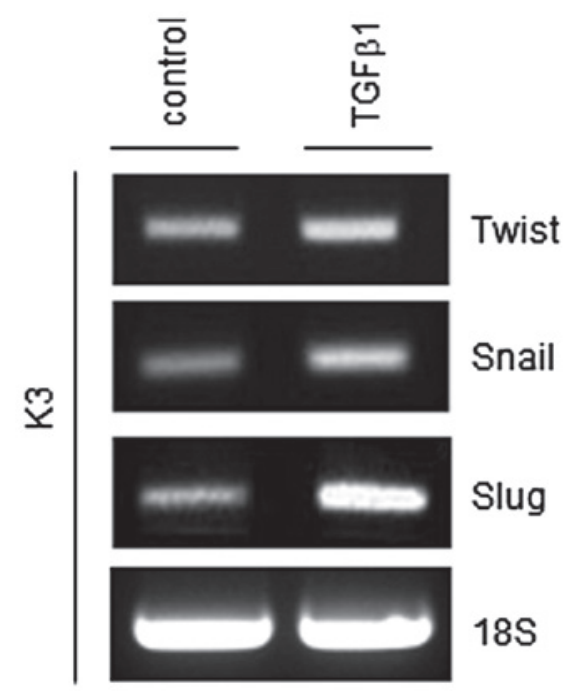

Figure 4. TGF $\beta 1$ treatment increases transcript levels of the epithelial-mesenchymal transition mediators Twist, Snail and Slug. TGF $\beta 1$, transforming growth factor $\beta$.

HNSCC CSCs, indicating that TGF $\beta 1$ may enhance the chemoresistance of HNSCC CSCs through increased expression of ABCG2 (Fig. 3B).
A

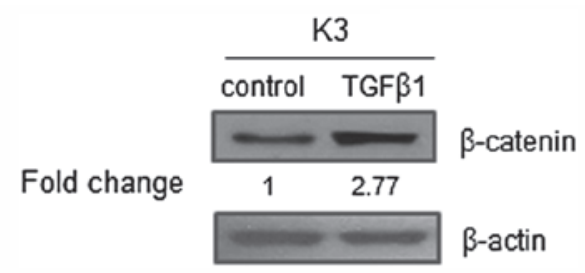

B
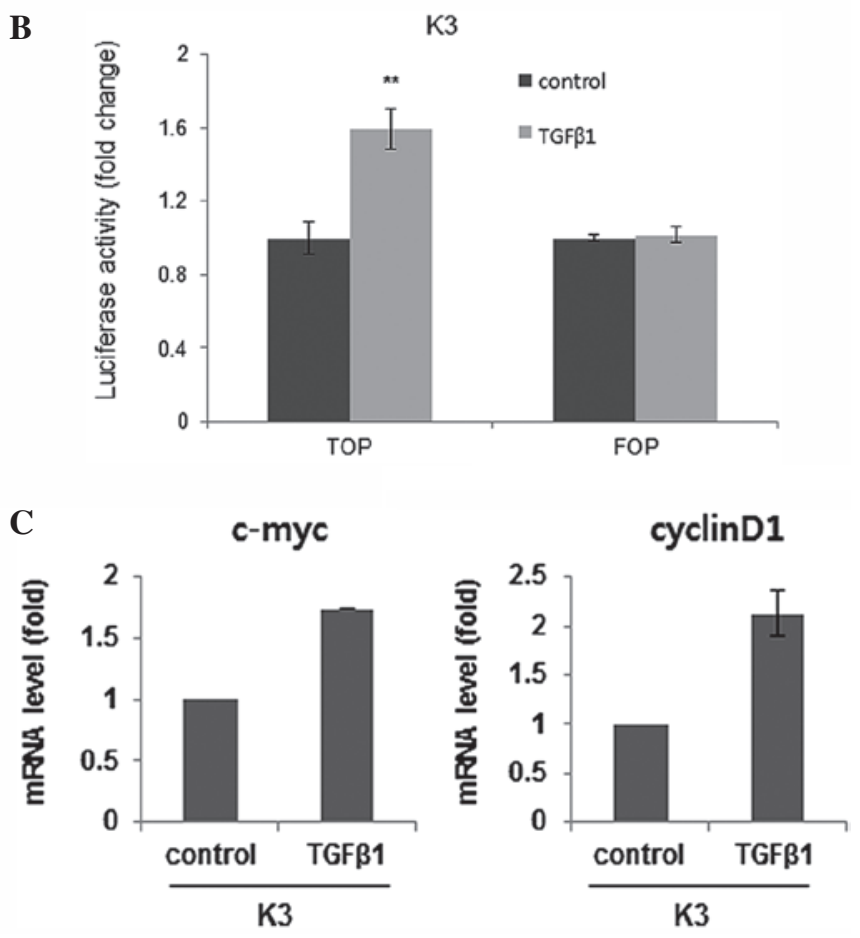

Figure 5. TGF $\beta 1$ treatment activates the canonical Wnt signaling pathway. (A) Protein level of $\beta$-catenin in TGF $\beta 1$ - or control (DMSO)-treated HNSCC CSCs. (B) TOP/FOP luciferase activity in TGF $\beta 1$ - or control (DMSO)-treated HNSCC CSCs. ${ }^{* *} \mathrm{P}<0.01$. (C) Transcript levels of $\beta$-catenin target genes (c-Myc and cyclin D1) in TGF 1 1- or control (DMSO)-treated HNSCC CSCs. TGF $\beta 1$, transforming growth factor $\beta$; HNSCC, head and neck squamous cell carcinoma; CSCs, cancer stem cells; DMSO, dimethyl sulfoxide; TOP, optimal Tcf-binding site; FOP, far-from-optimal Tcf-binding site

TGF $\beta 1$ increases the expression of epithelial-mesenchymal transition (EMT) regulators. EMT is a key process in tumor invasion and metastasis (15). Previously, it was reported that the EMT process can generate cancer cells with a stem cell phenotype (15). Thus, whether TGF $\beta 1$ can increase central regulators of the EMT process, such as Twist, Snail and Slug, was investigated. As shown in Fig. 4, mRNA expression levels of all three EMT regulators were increased in TGF $\beta 1$-treated HNSCC CSCs compared with control-treated HNSCC CSCs.

TGF $\beta 1$ activates canonical Wnt $/ \beta$-catenin signaling. It was previously suggested that $\mathrm{Wnt} / \beta$-catenin contributes to the stemness of HNSCC cells (16). Thus, whether TGF $\beta 1$ acts as an upstream stimulator of $\mathrm{Wnt} / \beta$-catenin signaling was investigated. Administration of TGF $\beta 1$ increased the expression of $\beta$-catenin, which is an effector of the Wnt pathway (Fig. 5A). Also, TGF $\beta 1$ treatment increased the activity of a Wnt/ $\beta$-catenin-dependent reporter as a functionally more relevant indicator (Fig. 5B). Furthermore, the transcript levels of Wnt/ $\beta$-catenin signaling target genes, such as the c-myc and cyclin D1 genes, were increased in TGF 31 -treated HNSCC 
CSCs (Fig. 5C). Overall, these data suggest that TGF $\beta 1$-induced stemness may be associated with Wnt pathway upregulation in HNSCC CSCs.

\section{Discussion}

Numerous previous studies have indicated that a rare population of cells within the entire tumor bulk, termed CSCs or cancer-initiating cells, possess capabilities for tumor initiation and progression, and in certain cases, metastasis, which are not found in the majority of cells $(5,14,17)$. In clinical settings, these cells exhibit an intrinsic resistance to popular chemotherapeutic agents, preventing complete eradication of the tumor cells following treatment. Therefore, an understanding of the molecular mechanism of CSCs as a treatment target is critical for the design and development of effective anticancer therapies against tumor relapse.

Several signal transmission pathways regulating CSC pathobiology have been suggested, including the Wnt, Notch and $\mathrm{Hh}$ pathways (18). Previously, members of the TGF $\beta$ cytokine family, such as TGF $\beta \mathrm{s}$, bone morphogenetic proteins (BMPs), Nodal and activins, were also shown to be involved in the control of the CSC phenotype, particularly in glioblastoma multiforme (GM) $(19,20)$. Notably, BMP and TGF $\beta$ have conflicting effects on GM CSC proliferation, although they are included in the same family (TGF $\beta)(19,20)$. BMP has been shown to inhibit tumor growth and to induce differentiation of GM CSCs (19). By contrast, TGF $\beta$ promotes self-renewal and prevents differentiation, enhancing the oncogenic ability of GM CSCs (21).

TGF $\beta 1$ expression is observed in $\sim 80 \%$ of cases of human HNSCC and correlates with more advanced disease and reduced survival (11). In addition, TGF $\beta 1$ overexpression promotes tumorigenesis in a paracrine manner, leading to increased inflammation and angiogenesis (11). However, different TGF $\beta$ signaling disruptions also enhance epithelial carcinogenesis through various mechanisms and are common in HNSCC, suggesting biphasic roles for this signaling pathway in tumorigenesis of HNSCC (22). Also, there are extremely few previous studies regarding the effects of TGF $\beta$ ligands on HNSCC CSCs. Thus, the present study aimed to clarify the role of TGF $\beta 1$ in HNSCC CSC biology.

In the present study, it was shown that TGF $\beta 1$ treatment enhances self-renewal and stemness-associated genes (Oct4 and Sox2) expression in HNSCC CSCs. In addition, this treatment was shown to result in enrichment of the $\mathrm{ALDH}^{+}$cell population, expressing a putative marker of HNSCC CSCs. Furthermore, TGF $\beta 1$ treatment results in an increase of stem cell-like traits, such as resistance to conventional chemotherapy (cisplatin) for treatment of HNSCC patients. Collectively, the present data suggest that TGF $\beta 1$ may be involved in the regulation of cells with stem cell-like traits in HNSCC.

Mechanistically, it has been shown that the regulation of GM CSC self-renewal by TGF $\beta$ is mediated by leukemia inhibitory factor (LIF), a member of the interleukin 6 (IL6) family of cytokines, and by signaling through a heterodimeric receptor complex composed of the glycoprotein 130 and the LIF receptor inducing the Janus kinase-signal transducers and activators of transcription pathway (21). In addition, IL6 may also be a mediator of the induction of GM CSC self-renewal by TGF $\beta$ (23). Notably, the present study showed that TGF $\beta$ administration increased the expression of $\beta$-catenin, a major effector of the canonical Wnt signal, although a detailed mechanism is not presented here. Serra et al observed that Wnt5a, a Wnt ligand, was directly regulated by TGF $\beta$ in primary mammary gland cells, and they identified Smad binding sites in the Wnt5a promoter (24). Thus, crosstalk between TGF $\beta$ signaling and Wnt signaling may be significant in HNSCC CSC biology. Additional studies are required to show how TGF $\beta$ signaling interacts with Wnt signaling.

Based on these data, the present findings demonstrate that TGF $\beta 1$ may have an important role in HNSCC CSC genesis and that its inhibitors may be valuable for eradicating HNSCC CSCs.

\section{Acknowledgements}

This study was supported by Konkuk University Hospital (grant no., 201403).

\section{References}

1. Jemal A, Murray T, Samuels A, Ghafoor A, Ward E and Thun MJ: Cancer statistics, 2003. CA Cancer J Clin 53: 5-26, 2003.

2. Forastiere A, Koch W, Trotti A and Sidransky D: Head and neck cancer. N Engl J Med 345: 1890-1900, 2001.

3. Yamano Y, Uzawa K, Saito K, Nakashima D, Kasamatsu A, Koike H, Kouzu Y, Shinozuka K, Nakatani K, Negoro K, et al: Identification of cisplatin-resistance related genes in head and neck squamous cell carcinoma. Int J Cancer 126: 437-449, 2010.

4. Haddad RI and Shin DM: Recent advances in head and neck cancer. N Engl J Med 359: 1143-1154, 2008.

5. Lim YC, Oh SY, Cha YY, Kim SH, Jin X and Kim H: Cancer stem cell traits in squamospheres derived from primary head and neck squamous cell carcinomas. Oral Oncol 47: 83-91, 2011.

6. Mannelli G and Gallo O: Cancer stem cells hypothesis and stem cells in head and neck cancers. Cancer Treat Rev 38: 515-539, 2012.

7. Liu L, Jiao J, Wang Y, Wu J, Huang D, Teng W and Chen L: TGF-betal gene polymorphism in association with diabetic retinopathy susceptibility: A systematic review and meta-analysis. PLoS One 9: e94160, 2014.

8. White RA, Malkoski SP and Wang XJ: TGF $\beta$ signaling in head and neck squamous cell carcinoma. Oncogene 29: 5437-5446, 2010.

9. Siegel PM and Massagué J: Cytostatic and apoptotic actions of TGF-beta in homeostasis and cancer. Nat Rev Cancer 3: 807-821, 2003.

10. Lu SL, Herrington H, Reh D, Weber S, Bornstein S, Wang D, Li AG, Tang CF, Siddiqui Y, Nord J, et al: Loss of transforming growth factor-beta type II receptor promotes metastatic head-and-neck squamous cell carcinoma. Genes Dev 20: 1331-1342, 2006.

11. Lu SL, Reh D, Li AG, Woods J, Corless CL, Kulesz-Martin M and Wang XJ: Overexpression of transforming growth factor beta1 in head and neck epithelia results in inflammation, angiogenesis, and epithelial hyperproliferation. Cancer Res 64: 4405-4410, 2004.

12. You H, Ding W and Rountree CB: Epigenetic regulation of cancer stem cell marker CD133 by transforming growth factor-beta. Hepatology 51: 1635-1644, 2010.

13. Kashyap V, Rezende NC, Scotland KB, Shaffer SM, Persson JL, Gudas LJ and Mongan NP: Regulation of stem cell pluripotency and differentiation involves a mutual regulatory circuit of the NANOG, OCT4, and SOX2 pluripotency transcription factors with polycomb repressive complexes and stem cell microRNAs. Stem Cells Dev 18: 1093-1108, 2009.

14. Zou B, Sun S, Qi X and Ji P: Aldehyde dehydrogenase activity is a cancer stem cell marker of tongue squamous cell carcinoma. Mol Med Rep 5: 1116-1120, 2012.

15. Kalluri R and Weinberg RA: The basics of epithelial-mesenchymal transition. J Clin Invest 119: 1420-1428, 2009. 
16. Lee SH, Koo BS, Kim JM, Huang S, Rho YS, Bae WJ, Kang HJ, Kim YS, Moon JH and Lim YC: Wnt/ $\beta$-catenin signalling maintains self-renewal and tumourigenicity of head and neck squamous cell carcinoma stem-like cells by activating Oct4. J Pathol 234: 99-107, 2014.

17. Dalerba P and Clarke MF: Cancer stem cells and tumor metastasis: First steps into uncharted territory. Cell Stem Cell 1: 241-242, 2007

18. Garcia Campelo MR, Alonso Curbera G, Aparicio Gallego G, Grande Pulido E and Antón Aparicio LM: Stem cell and lung cancer development: Blaming the Wnt, Hh and Notch signalling pathway. Clin Transl Oncol 13: 77-83, 2011.

19. Piccirillo SG, Reynolds BA, Zanetti N, Lamorte G, Binda E, Broggi G, Brem H, Olivi A, Dimeco F and Vescovi AL: Bone morphogenetic proteins inhibit the tumorigenic potential of human brain tumour-initiating cells. Nature 444: 761-765, 2006.
20. Lee J, Son MJ, Woolard K, Donin NM, Li A, Cheng CH, Kotliarova S, Kotliarov Y, Walling J, Ahn S, et al: Epigenetic-mediated dysfunction of the bone morphogenetic protein pathway inhibits differentiation of glioblastoma-initiating cells. Cancer Cell 13: 69-80, 2008.

21. Seoane J: TGFbeta and cancer initiating cells. Cell Cycle 8: 3787-3788, 2009.

22. Levy L and Hill CS: Alterations in components of the TGF-beta superfamily signaling pathways in human cancer. Cytokine Growth Factor Rev 17: 41-58, 2006.

23. Wang H, Lathia JD, Wu Q, Wang J, Li Z, Heddleston JM, Eyler CE, Elderbroom J, Gallagher J, Schuschu J, et al: Targeting interleukin 6 signaling suppresses glioma stem cell survival and tumor growth. Stem Cells 27: 2393-2404, 2009.

24. Serra R, Easter SL, Jiang W and Baxley SE: Wnt5a as an effector of TGF $\beta$ in mammary development and cancer. J Mammary Gland Biol Neoplasia 16: 157-167, 2011. 